\title{
Functional Convergence of Dopaminergic and Cholinergic Input Is Critical for Hippocampus-Dependent Working Memory
}

\author{
Liselijn A. B. Wisman, ${ }^{1}$ Gurdal Sahin, ${ }^{1}$ Matthew Maingay, ${ }^{1}$ Giampiero Leanza, ${ }^{2 *}$ and Deniz Kirik ${ }^{1 *}$ \\ ${ }^{1}$ Brain Repair and Imaging in Neural Systems, Department of Experimental Medical Science, Lund University, 22184 Lund, Sweden, and 2 B.R.A.I.N. Centre \\ for Neuroscience, Department of Life Sciences, University of Trieste, 34127 Trieste, Italy
}

\begin{abstract}
Although Parkinson's disease is a movement disorder, in many patients cognitive dysfunction is an important clinical sign. It is not yet clear whether this is attributable solely to a decrease in dopamine levels, or whether other neurotransmitter systems might be involved as well. In the present study, the importance of the mesocorticolimbic dopamine pathway and a possible convergence with forebrain cholinergic projections to neocortex and hippocampus in the regulation of learning and memory abilities were investigated by using specific lesion paradigms in one or both systems. Lesioning of dopaminergic neurons in the ventral tegmental area resulted in an impaired performance in the reference memory task, whereas the execution of the working memory tasks appeared to be unaffected in the Morris water maze. Analysis of the swim paths revealed that the dopamine-depleted animals were capable of adapting a search strategy on a given testing day but failed to transfer this information to the next day, suggesting a deficit in information storage and/or recall. In contrast, cholinergic lesions alone were without effect in all test paradigms. However, when both dopamine and acetylcholine were depleted, animals were also impaired in the working memory task, indicating that a functional convergence of the inputs from these systems was critical for acquisition of spatial memory. Interestingly, such an additional acquisition deficit appeared only after hippocampal cholinergic depletion regardless of a concurrent disruption of basalocortical cholinergic afferents. Thus, further analyses of cholinergic alterations may prove useful in better understanding the cognitive symptoms in Parkinson's disease.
\end{abstract}

Key words: Parkinson's disease; dopamine; acetylcholine; 6-hydroxydopamine; 192 IgG-saporin; learning and memory; water maze test; stereology; rat

\section{Introduction}

Parkinson's disease (PD) is clinically characterized by motor dysfunction, and it is defined pathologically by a prominent loss of dopamine (DA)-synthesizing neurons in the substantia nigra (SN) (Hirsch et al., 1988; Fearnley and Lees, 1991). However, even in early stages there is a marked clinical heterogeneity, which is in part attributable to the occurrence of cognitive impairments (Foltynie et al., 2002; Lewis et al., 2005). In general, the incidence of dementia among PD patients is two to three times higher than in age-matched controls (Marder et al., 1995; Aarsland et al., 2003). Approximately $30-50 \%$ of the PD patients develop dementia during a 4-8 year follow-up period (Aarsland et al., 2003; Williams-Gray et al., 2007), and the risk of developing dementia increases as the disease progresses (Braak et al., 2005).

Received Feb. 24, 2008; revised June 11, 2008; accepted June 18, 2008.

This work was supported by Swedish Research Council Grants 2003-33P-14778-01A and 2003-33X-14552-03A (D.K.), Konung Gustav 5th och Drottning Victorias Stiftelse (D.K.), and the Kathleen Foreman-Casali Foundation (G.L.). L.A.B.W. was supported by a fellowship from The Nordic Center of Excellence Programme in Molecular Medicine-Neurodegeneration. We thank Anneli Josefsson, Ulla Jarl, and Bengt Mattsson for expert technical help. *G.L. and D.K. contributed equally to this work.

Correspondence should be addressed to Deniz Kirik, Brain Repair and Imaging in Neural Systems, Department of Experimental Medical Science, Lund University, BMC D11, SE-22184 Lund, Sweden. E-mail: Deniz.Kirik@med.lu.se. M. Maingay's present address: Quintiles Ltd., 49-51 Symonds Street, Auckland 1010, New Zealand. D0I:10.1523/JNEUROSCI.1885-08.2008

Copyright $\odot 2008$ Society for Neuroscience $\quad$ 0270-6474/08/287797-11\$15.00/0
Impairment of executive functions and working memory appear to be major features of parkinsonian dementia. Because the main lesion in PD patients is loss of dopaminergic neurons in the nigrostriatal pathway, it has been suggested that the observed cognitive deficits may be related to a loss of DA in the caudate nucleus, which in turn results in the disruption of frontostriatal circuitry (Owen et al., 1998; Dagher et al., 2001; Lewis et al., 2003). Nevertheless, cognitive disturbances in PD patients may also be associated to alterations in mesocorticolimbic DA input. In fact, loss of DA neurons in the ventral tegmental area (VTA) is a well-recognized feature of PD (Scatton et al., 1983; Damier et al., 1999). Furthermore, imaging studies provided evidence that a direct loss of cortical DA could compromise executive function in PD patients (Cools et al., 2002; Mattay et al., 2002).

Alternatively, but not necessarily in contrast, the cognitive decline observed in PD patients might also be associated to dysfunction of nondopaminergic neuronal populations (Agid et al., 1987). Among these, cholinergic neurons in the medial septum (MS), the diagonal band of Broca (DBB), and the nucleus basalis magnocellularis (NBM) innervating the entire cortical mantle, the olfactory bulb, the hippocampus, and the amygdala appear to be of particular interest (for review, see Woolf, 1991). In fact, in addition to their implication in the cognitive decline observed in Alzheimer's disease patients (Bartus et al., 1982; Coyle et al., 
1983), there is evidence indicating that cholinergic deficits are present also in PD patients. Thus, a dramatic $45 \%$ reduction in choline acetyltransferase (ChAT) activity in the frontal cortex (Dubois et al., 1983) and a 60\% loss of cholinergic neurons in the NBM (Nakano and Hirano, 1984) have been reported in PD patients compared with age-matched controls. Whether similar reductions in ChAT activity occur also in the hippocampal formation is not known. Together, these observations are compatible with a cooperative role between dopaminergic and cholinergic mechanisms in cognition; however, this issue has not been sufficiently explored thus far.

In the present study, we sought to investigate whether a possible functional interaction between dopaminergic and cholinergic systems might be required for the regulation of cognitive abilities in spatial memory tasks. Toward this end, we used a specific lesioning paradigm, in which dopaminergic neurons in the VTA and cholinergic neurons in the MS and vertical limb of the $\mathrm{DBB}(\mathrm{MS} / \mathrm{vDBB})$ and/or NBM giving rise to projections to the frontal cortex and/or hippocampus were selectively targeted by specific neurotoxins. A range of behavioral measures, in conjunction with histological and biochemical indices of neuronal and terminal fiber loss, were then used to assess the anatomical and functional consequences of the lesions.

\section{Materials and Methods}

Animals. A total of 169 young adult female Sprague Dawley rats (B\&K Universal) were used in this study. The animals were 8-10 weeks old and weighed $\sim 225 \mathrm{~g}$ at the beginning of the experiment. All animals were housed under a $12 \mathrm{~h}$ light/dark cycle with ad libitum access to food and water, except during the paw-reaching task when the animals were on a restricted diet as described below. All procedures were approved by the Ethical Committee for the Use of Laboratory Animals at Lund-Malmö region.

The animals were allocated into the following groups: intact controls ( $n=21$ for behavioral studies; $n=6$ for biochemical analysis); vehicle controls $(n=8)$, which were injected with $0.05 \%$ ascorbate saline into the VTA in combination with PBS into the NBM $(n=4)$ or the MS/vDBB $(n=4)$; animals injected with 192 IgG-saporin toxin into the $\mathrm{NBM}(n=$ 19); animals injected with $192 \mathrm{IgG}$-saporin immunotoxin into the MS/ $\operatorname{vDBB}(n=15)$; or animals injected with 6-hydroxydopamine (6OHDA) toxin into VTA ( $n=21$ for behavioral analysis; $n=4$ for biochemical analysis). Additional animals underwent combined injections of the toxins into the respective regions so as to form the NBM+MS/ $\operatorname{vDBB}$ group $(n=20)$, the VTA+NBM group $(n=15)$, the VTA+MS/ $\operatorname{vDBB}$ group $(n=30)$, and the VTA $+\mathrm{NBM}+\mathrm{MS} / \operatorname{vDBB}$ group $(n=20)$. To ensure consistency, the lesion surgeries and behavioral testing were performed in four subsequent sessions in that animals belonging to several of the groups detailed above were included in each session. All sessions included naive or vehicle-treated control animals $(n=3-8$ in different sessions).

Experimental design and inclusion criteria. After surgery the animals were given $4-5$ weeks to recover before they underwent sequential behavioral analyses. First, spatial learning and memory were evaluated by the Morris water maze (MWM) test over a period of 2 weeks using specific designs enabling the detection of reference and working memory deficits. Afterward, the striatum-dependent learning was tested with the paw-reaching task for another 2 week period, followed by an open-field activity test to assess spontaneous and apomorphine-induced locomotor activity. On completion of all behavioral tests, at 11-12 weeks after surgery, the animals were killed, and the brains were processed for immunohistochemistry to assess the extent of the dopaminergic and cholinergic lesions. Specific lesions of the VTA neurons were obtained by local injection of 6-OHDA, as described previously (Maingay et al., 2006). With such a lesioning paradigm, and provided that neuronal loss exceeds $40 \%$, a consistent locomotor response to apomorphine stimulation is observed, reminiscent of a mesocortical limbic dopaminergic dysfunc- tion. Thus, the presence of a $\geq 40 \%$ depletion of TH-positive neurons in the VTA was taken as a criterion for inclusion in this study. As to the cholinergic lesions, the dose of immunotoxin and the injection sites to target cholinergic neurons in the basal forebrain (BF) were carefully selected on the basis of pilot experiments. The production of a consistent $\geq 60 \%$ loss of ChAT-immunoreactive neurons in the MS/vDBB, the NBM, or both of these nuclei was taken as a criterion for inclusion. To analyze the DA values in the prefrontal cortex and hippocampus, four animals from the VTA group and six animals from the control group were killed for HPLC measurements as described below.

The adoption of the above criteria resulted in the following group sizes: intact controls $(n=19)$, vehicle controls $(n=8)$, NBM $(n=14)$, $\mathrm{MS} / \mathrm{vDBB}(n=15), \mathrm{NBM}+\mathrm{MS} / \mathrm{vDBB}(n=14), \mathrm{VTA}(n=15)$, $\mathrm{VTA}+\mathrm{NBM}(n=9), \mathrm{VTA}+\mathrm{MS} / \mathrm{vDBB}(n=11)$, and VTA + NBM+MS/ $\operatorname{vDBB}(n=11)$.

Surgical procedures. All surgical procedures were done under $2 \%$ isofluorane anesthesia (Schering-Plough Animal Health). The animals were placed in a stereotaxic frame, and an incision was made on the skin above the skull. The injection sites were determined by anteroposterior (AP) and mediolateral (ML) coordinates, calculated from bregma, whereas the dorsoventral (DV) coordinates were calculated from the dura (all in millimeters) according to the stereotaxic atlas of Paxinos and Watson (2007). A $5 \mu \mathrm{l}$ Hamilton syringe fitted with a glass pipette (outer diameter, $60-80 \mu \mathrm{m}$ ) was used for the injections. To target the mesocorticolimbic dopaminergic projection neurons, $2 \mu \mathrm{l}$ of 6-OHDA $(4.5 \mu \mathrm{g} / \mu \mathrm{l}$ in $0.05 \%$ ascorbate saline; Sigma-Aldrich) was injected in the VTA (AP, $-5.5 ; \mathrm{ML},-1.6 ; \mathrm{DV},-7.3$ ) with an angle of $13^{\circ}$ in the coronal plane. The coordinates and the injection parameters for this lesion paradigm were optimized in a previous study (Maingay et al., 2006). Cholinergic neurons in the NBM and the MS/vDBB were lesioned by bilateral injection of the immunotoxin 192 IgG-saporin (lot no. 41-105; Advanced Targeting Systems). Briefly, $0.85 \mu \mathrm{l}$ of the immunotoxin (dissolved at a concentration of $0.142 \mu \mathrm{g} / \mu \mathrm{l}$ in PBS) was injected bilaterally (AP, -0.5 ; ML, \pm 1.5 ; $\mathrm{DV},-4.5$ ) to target the NBM, whereas the cholinergic neurons in the MS/vDBB were lesioned by injection of a total of $0.5 \mu 1192$ IgG-saporin $[0.22 \mu \mathrm{g} / \mu \mathrm{l}(\mathrm{AP}, 0.5 ; \mathrm{ML}, \pm 0.6)]$ divided into two deposits [DV, -7.7 $(0.3 \mu \mathrm{l})$ and $\mathrm{DV},-6.1(0.2 \mu \mathrm{l})]$ on each side of the brain. In a separate set of experiments, we have observed that injection of the same lot of the toxin at the same dose and coordinates as above caused no detectable toxicity to parvalbumin-expressing neurons in the MS/vDBB (G. Leanza, G. Capodieci, and L. Arancio, unpublished observations).

Behavioral tests. The performance of the lesioned and control animals was assessed using three well-established behavioral tasks that were administered in sequence.

The Morris water maze task was used to test spatial learning and memory. The apparatus consisted of a large circular tank ( $180 \mathrm{~cm}$ in diameter) filled with water made opaque by the addition of nontoxic paint. The tank was placed in a room containing extramaze cues that can be used for orientation. Four points conventionally indicated as north $(\mathrm{N})$, south $(\mathrm{S})$, east $(\mathrm{E})$, and west $(\mathrm{W})$ were used as starting positions and divided the tank into four quadrants. A circular platform $(15 \mathrm{~cm}$ in diameter) was anchored to the bottom of the tank in the center of the SW quadrant with its top $2 \mathrm{~cm}$ below the water surface so as to be invisible from within the pool. Four annuli were defined as a circular area in the middle of each quadrant, corresponding to the site where the platform would have been if placed in that quadrant.

Two different testing paradigms, specifically designed to evaluate reference and working memory abilities, were used in sequence. In the reference memory version of the test, the platform was kept in the same position, and the rats were given four trials per day over 7 consecutive days. In each trial, the rats were released from a different starting point and given $60 \mathrm{~s}$ to find the platform and climb onto it. A 20 s intertrial time was permitted. The latency to locate the platform, the total distance swum, and the average swim speed were recorded using a video tracking system (EthoVision 3.0.13; Noldus). After the last trial on day 7, the platform was removed from the tank, and a final spatial probe trial was given to all animals. In this latter trial, the rats were allowed to swim ad libitum for $60 \mathrm{~s}$, during which the time spent, distance swum, and the number of annulus crossings in each quadrant were recorded. On the 
following day, the working memory version of the test was administered using a testing paradigm adapted from Lehmann et al. (2000). The training was conducted over 4 consecutive days; the animals were released from the same starting point in each of the four daily trials. The platform position was changed everyday but kept constant over the four trials on each day. On day 1 , the platform position was in the center of the NE quadrant, whereas the release point was in the middle of the SW quadrant at the edge. On day 2, the platform position was in the center of the SE quadrant, whereas the release point was in the middle of the NE quadrant. The platform position was in the center of the NW quadrant, whereas the release point was the middle of the SE quadrant on day 3. On the last day, the position of the platform was at the edge of the NW and SW quadrant, whereas the release point was at north. The latency and distance swum, required to locate the hidden platform, were recorded as above. The difference between latency scores on trials 2 and 3 , expressed as percentage of trial 1, provided an additional measure of working memory performance ("savings") (see also Netto et al., 1993). The same analysis was also performed on animals' performance between trials 3 and 4 to rule out the possible adoption of unrelated across-day trajectory strategies that would be more dependent on striatal, rather than hippocampal, mechanisms (see also White, 1997; Jog et al., 1999; Cassel et al., 2002).

To evaluate possible differences in the search strategy adopted by the rats during the execution of the working memory task, an observer blinded to the identity of the animals analyzed the swim paths from the control, VTA, and VTA+MS/vDBB groups. Close inspection of swim paths revealed three main patterns of search behavior: (1) use of a spatial search strategy. This pattern occurred when the animal was efficiently using the extramaze cues, and it was inferred by the frequent and sharp direction changes in the swim path. (2) Use of a nonspatial search strategy. This pattern occurred when the animal used egocentric cues, rather than extramaze (i.e., allocentric) cues. Typically, this behavior was illustrated by a complex, albeit poorly focused, circular swimming that might nevertheless help to locate the platform. (3) No apparent use of any search strategy. Such pattern was illustrated by the frequent adaptation of a thigmotaxic swimming, whereby the animal swam in a circular manner close to or in contact with the wall of the tank.

The paw-reaching task (Montoya et al., 1991) was used to assess the striatum-dependent motor learning as previously described (Kirik et al., 1998). Briefly, rats were deprived of their regular food starting $2 \mathrm{~d}$ before the testing period. During this period, the sugar pellets used in this test (Test Diet; Sandown Scientific) were introduced in the home cages to familiarize the animals. On the first testing day, the animals were placed in narrow Plexiglas boxes holding a central platform and staircases with seven levels on both sides. Ten sugar pellets were presented on each one of the four baited stairs (levels 2-5) bilaterally. The animals were allowed to reach and retrieve the pellets for $20 \mathrm{~min}$. At the end of the session, the number of pellets found on any staircase level, as well as the pellets dropped to the bottom reservoir, was counted to calculate the pellets removed from each stair (attempts) and the total number of pellets missed (errors). The difference between the two measures constituted the total number of pellets eaten (success). Animals were tested for 9 consecutive days until a plateau performance was obtained in the successful retrievals. As a dependent variable for statistical analysis, we used the percentage of errors made compared with the number of attempts.

Open-field activity test was used to assess horizontal locomotor activity. The apparatus consisted of $40 \times 40 \times 38 \mathrm{~cm}$ Plexiglas boxes equipped with $16 \times 16$ photobeam systems automatically controlled by the FlexField Software (San Diego Instruments). The test was conducted over $3 \mathrm{~d}$. The animals were habituated for $1 \mathrm{~h}$ sessions on the first $2 \mathrm{~d}$. On the third day, baseline activity was recorded for $1 \mathrm{~h}$; the animals then received a subcutaneous injection of a low dose of apomorphine $(0.1 \mathrm{mg} / \mathrm{kg}$ in $0.02 \%$ ascorbic acid) before being monitored for an additional $2 \mathrm{~h}$. As a dependent variable for statistical analysis, the total amount of beam interruptions in the first $40 \mathrm{~min}$ after an apomorhine injection was used.

HPLC analysis. The tissue content of DA and its metabolites, homovanillic acid and 3,4-dihydroxyphenylacetic acid, was assessed in VTA lesioned and control animals. After decapitation, the brains were rapidly removed, and the prefrontal cortex and hippocampus were dissected out, frozen on dry ice, and stored at $-80^{\circ} \mathrm{C}$. At the time of analysis, tissue samples were homogenized in $0.1 \mathrm{M}$ perchloric acid and centrifuged at $10,000 \mathrm{rpm}$ for $10 \mathrm{~min}$ before being filtered through minispin filters and spun down for an additional $3 \mathrm{~min}$ at 10,000 rpm. The tissue extracts were then analyzed by HPLC as described previously (Maingay et al., 2006). Briefly, $20 \mu \mathrm{l}$ of each sample was injected by a cooled autosampler (Midas) into an an electrochemical detector (Coulochem III; ESA Biosciences) equipped with a guard cell and an analytical cell. The guard cell was set at $400 \mathrm{mV}$, and electrode 2 was set as at $-350 \mathrm{mV}$. The mobile phase (sodium acetate, $5 \mathrm{~g} / \mathrm{L} ; \mathrm{Na}_{2}$ EDTA, $30 \mathrm{mg} / \mathrm{L}$; octane-sulfonic acid, $100 \mathrm{mg} / \mathrm{L}$; methanol, $9 \%$; $\mathrm{pH} 4.2$ ) was delivered at a flow rate of 500 $\mu \mathrm{l} / \mathrm{min}$ to a reversed-phase $\mathrm{C} 18$ column $(4.0 \times 100 \mathrm{~mm}$; Chromtech $)$. The peaks were quantitatively analyzed using the Clarity Chromatographic Station (DataApex). Tissue DA content in the VTA group was analyzed in animals with clear-cut neuronal loss in the VTA, as confirmed by histology (see below).

Histological analysis. On completion of the behavioral tests, the animals were killed by using an overdose of pentobarbital and perfused transcardially with $50 \mathrm{ml}$ of saline followed by $250 \mathrm{ml}$ of $4 \%$ paraformaldehyde in $0.1 \mathrm{~m}$ phosphate buffer $(\mathrm{PB})(\mathrm{pH} 7.4)$. The brains were dissected free from the skull and were postfixed for an additional $2 \mathrm{~h}$ in the same fixative. Subsequently, the brains were cryoprotected in $25 \%$ sucrose in $\mathrm{PB}$ at $4^{\circ} \mathrm{C}$, and sections were cut into six series on a freezing microtome at $35 \mu \mathrm{m}$ thickness.

Immunohistochemical stainings for phenotypic markers of dopaminergic and cholinergic cells were performed on free-floating sections. All incubations were performed in potassium $\mathrm{PB}(\mathrm{KPB})$, and the sections were rinsed three times between each incubation step using the same buffer. Sections were first quenched in $3 \% \mathrm{H}_{2} \mathrm{O}_{2}$ and $10 \%$ methanol. The nonspecific binding sites were then blocked by using $5 \%$ normal horse serum (NHS) and $0.25 \%$ Triton X-100 in KPB. Subsequently, the sections were incubated with a primary antibody in 5\% NHS/0.25\% Triton $\mathrm{X}-100$. To visualize dopaminergic neurons, sections were incubated overnight with a 1:1000 dilution of the mouse anti-TH antibody (MAB318; Millipore Bioscience Research Reagents). For the identification of cholinergic neurons, a 1:500 dilution of the mouse anti-ChAT antibody (MAB305; Millipore Bioscience Research Reagents) was used, and the sections were incubated for $72 \mathrm{~h}$ at $4^{\circ} \mathrm{C}$. This was followed by incubation with the secondary biotinylated horse anti-mouse antibody (BA2001; Vector Laboratories). Subsequently, the sections were incubated with avidin-biotin-peroxidase complex (ABC-elite kit; Vector Laboratories). The reaction product was visualized using 3,3diaminobenzidine as a chromogen. Sections were mounted on chromealum-coated slides, dehydrated in ascending alcohol concentrations, cleared in xylene, and coverslipped in Depex (VWR International).

Stereological quantification. Stereology provides a reliable method for achieving unbiased estimates of the total number of neurons in a given brain region (Gundersen et al., 1988; West et al., 1991; West, 1999). In the present study, separate stereological analyses were conducted on ChATand TH-immunoreactive neurons in the basal forebrain nuclei and VTA, respectively, to assess the effects of the lesion treatment on these populations. For the basal forebrain nuclei, selection criteria were used as described previously (Fischer et al., 1989). Briefly, the MS/vDBB was defined as extending from the level of the genu of the corpus callosum rostrally, to the crossing of the anterior commissure caudally, setting the lateral edge of the vDBB at the medial border of the olfactory tubercle. The NBM was defined as a region rich in magnocellular cholinergic neurons located between the internal capsule and the globus pallidus, and extending from the level of the caudal septum rostrally, to the tail of the caudate-putamen caudally (Lehmann et al., 1980; Bigl et al., 1982). The lateral border of the VTA was defined by a vertical line passing through the medial tip of the cerebral penduncle and by the medial terminal nucleus of the accessory nucleus of the optic tract when present in the section. The dorsal tips of the mammillary and interfascicular nuclei defined the ventral border, and the ventral tip of the red nucleus was used as the exclusion border dorsally. The TH+ cells in the VTA were counted from the rostral SN until the end of pars reticulata, thus excluding the $\mathrm{TH}+$ neurons in the retrorubral area from the analysis (Maingay et al., 2006; Breysse et al., 2007). 
Random sampling was done using newCAST in the Visiopharm Integrator System software version 2.12.3.0 on a Nikon Eclipse 90i microscope using a $60 \times$ magnification oil lens with a numerical aperture of 1.40. Briefly, the NBM, $\mathrm{MS} / \mathrm{vDBB}$, and VTA were outlined as described above. This yielded an average of eight sections throughout each structure in a series (one of six sections in the brain). A counting frame was randomly placed on the first counting area and moved systematically over the entire inclusion area on each section. For all regions analyzed, estimations were performed bilaterally. The penetration of the TH and ChAT antibodies were determined by registration of the depth of each counted neuronal profile that appeared in focus within the counting frame. This analysis revealed an incomplete penetration of antibody, leaving 6-8 $\mu \mathrm{m}$ in the center poorly stained with the TH antibody and 4-6 $\mu \mathrm{m}$ with the ChAT antibody (Torres et al., 2006). The inclusion volume estimating total number of cells was therefore calculated, excluding this portion of the sections, and was done according to the optical fractionator principle (West, 1999).

Statistical analysis. Statistical comparisons were conducted using one-way ANOVA, twoway repeated-measures ANOVA one-sample $t$ test, or Pearson chi-square test, where appropriate. In cases in which the overall ANOVA indicated significant effects, post hoc analyses were conducted using either Tukey HSD or Dunnett's tests. The statistical significance was set at $p<0.05$. All statistical analyses were done using the JMP Statistical Software package version 5.0.1.2 (SAS Institute).

\section{Results}

The aim of the present study was to examine whether convergence of dopaminergic and cholinergic inputs to neocortical and/or hippocampal areas is required for maintenance of learning and memory functions. Therefore, the 6-OHDA or the 192 IgG-saporin neurotoxins were injected, either alone or in combination, to selectively target the mesocorticolimbic DA pathway or the septohippocampal and basalocortical cholinergic projections. The functional effects of the various lesioning treatments were studied using a range of well-established sensory-motor and cognitive tasks: the MWM test to evaluate reference and working memory performance, the paw-reaching performance to test striatumdependent motor learning ability, and apomophine-induced openfield activity as a measure of DA receptor supersensitivity.

Lesioning of the mesocorticolimbic DA pathway by 6-OHDA injection into the VTA resulted in an $\sim 50 \%$ loss of dopaminergic neurons, as estimated by stereological analysis of TH-positive cells in this nucleus (Fig. $1 E$ ). In contrast, TH-positive neurons in the SN were mostly unaffected (Fig. $1 A, B$ ). Measurements of DA levels by HPLC showed that there was an $83 \%$ reduction in the frontal cortex of VTA-lesioned animals $(35.7 \pm 11.6 \mathrm{fmol} / \mathrm{mg}$ tissue) compared with controls (210.2 $\pm 14.8 \mathrm{fmol} / \mathrm{mg}$ tissue). Similarly, in the hippocampus, DA levels were reduced by $\sim 74 \%$ after the VTA lesion (17.2 $\pm 11.6 \mathrm{fmol} / \mathrm{mg}$ tissue) compared with the control group $(65.2 \pm 13.8 \mathrm{fmol} / \mathrm{mg}$ tissue $)$. Using the same lesioning paradigm, we have shown previously that this level of partial neuronal loss can produce changes in locomotor activity
(Maingay et al., 2006). In fact, when the animals in the groups were injected with a low dose of apomorphine they exhibited a marked increase in activity over the 40 min testing period compared with intact animals (Fig. $1 F$ ). Cholinergic lesions did not affect the activity levels per se, nor did they appear to produce any modification of the responses elicited by the VTA lesion alone.

Cholinergic neurons in the MS/vDBB and the NBM regions were identified by ChAT immunohistochemistry and were observed as large cells with rich dendritic arborizations (Fig. 2A,E). Injection of $192 \mathrm{IgG}$-saporin into the MS/vDBB or the NBM produced a dramatic neuronal loss in the same regions (Fig. 2, compare $A, E$ with $B, F)$. Stereological analyses revealed $\sim 90 \%$ ChAT-positive cell loss in the MS/vDBB (Fig. $2 B, D, G$ ) and $\sim 80 \%$ neuronal depletion in the NBM (Fig. $2 F, H$ ). In previous studies it has been demonstrated that the IgG-saporin lesion paradigm does not affect the parvalbumin expressing neurons in MS/vDBB (Torres et al., 1994; Leanza et al., 1995).

VTA dopaminergic but not BF cholinergic neuronal cell loss induces acquisition deficits in the reference memory task

To assess spatial learning and memory the MWM test was used. The animals were first trained over 7 consecutive days on a ver- 


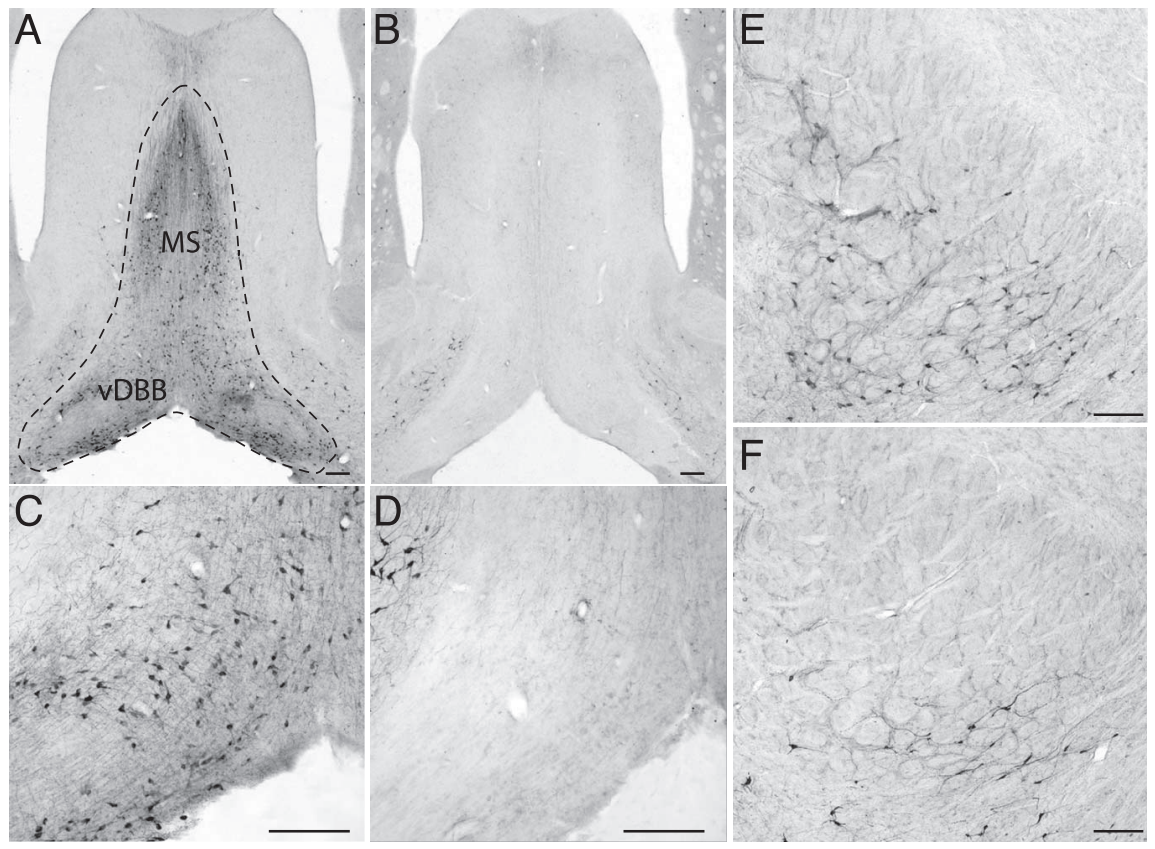

G ChAT+ neurons in the MS/vDBB

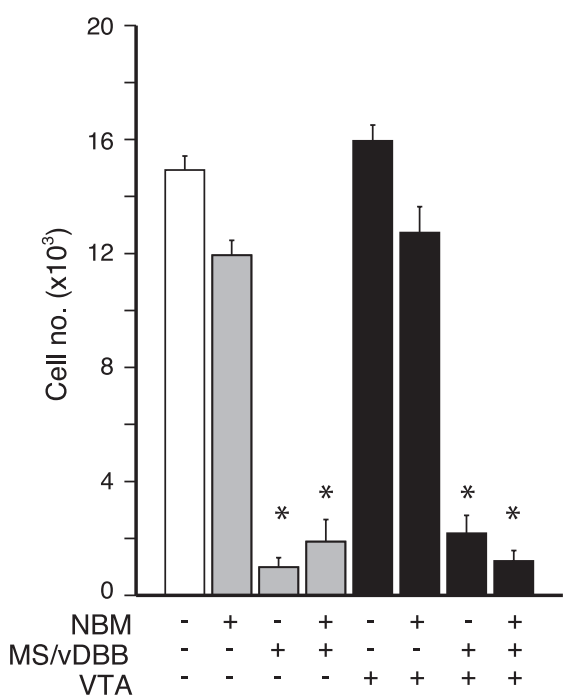

$\mathrm{H}$ ChAT+ neurons in the NBM

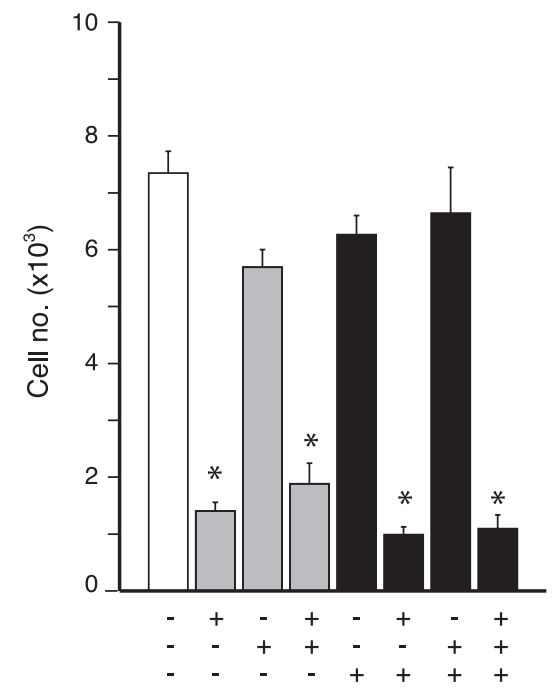

Figure 2. Stereological analysis was used to assess the extent of the cholinergic lesions in the different experimental groups. $\boldsymbol{A}-\boldsymbol{F}$, Cholinergic neurons were visualized with a ChAT antibody in the MS/vDBB $(\boldsymbol{A}-\boldsymbol{D})$ and $\operatorname{NBM}(\boldsymbol{E}, \boldsymbol{F}), \boldsymbol{G}, \boldsymbol{H}$, The lesion with 192 lgG-saporin resulted in $\sim 90 \%$ cholinergic cell loss in the $\operatorname{MS} / \mathrm{vDBB}(\boldsymbol{G})$ and $80 \%$ cell loss in the NBM $(\boldsymbol{H})\left(\boldsymbol{G}\right.$ : 1-way ANOVA, $F_{(7,109)}$ $=135.05, p<0.0001$, followed by a post hoc Tukey HSD test; $\boldsymbol{H}$ : 1 -way ANOVA, $F_{(7,109)}=49.82, p<0.0001$, followed by a post hoc Tukey HSD test). Scale bars, $200 \mu \mathrm{m}$. $\boldsymbol{G}, \boldsymbol{H}$, Groups are represented in the $x$-axis by presence ( + ) or absence ( - ) of a lesion in the given nucleus. Error bars indicate SEM.

sion of the task enabling the evaluation of reference memory abilities. In this design, the platform was maintained in a fixed position, and the animals were expected to learn to use the extra maze cues to locate the platform position efficiently and recall this information in the following test day. Because of practical reasons in surgical procedures and some of the behavioral tests, we have chosen to use female rats in the experiment. Although this might have caused an increased variance attributable to estrous cycle, it does not introduce any bias in the interpretation of the group differences. The overall performance in all groups and the relevant statistics are shown in Figure 3 and its legend. As expected, the animals in the control group rapidly learned to locate the platform and soon reached an asymptotic performance
(Fig. 3A) so that their latency scores on days 5-7 averaged $\sim 15 \mathrm{~s}$ (Fig. $3 B$ ). Lesioning the cholinergic neurons in the NBM, MS/vDBB, or both of these sites combined did not affect the rats' ability to find the platform. However, disruption of the forebrain DA input, induced by the VTA lesion, produced a marked impairment, seen as an increase in escape latency, regardless of the presence of a concurrent cholinergic depletion (Fig. $3 A, B$ ). The swim speed was recorded for all groups and did not differ between any of the treatment groups and the control animals ( $p>0.05$ for all comparisons). Therefore, essentially, the analysis of distance traveled gave the exact same group effects (ANOVA, $F_{(7,109)}=$ 49.53, $p<0.0001$; post hoc comparison with control group, $p<0.05$ for all VTAlesioned groups). Accordingly, in the free probe test the control and cholinergiclesioned groups were all actively searching for the platform at the usual position, whereas time spent in the training quadrant was reduced in the VTA-lesioned groups compared with the control group, suggestive of poorly-focused search behavior, which reached significance in the VTA, VTA+NBM, and VTA+MS/ $\mathrm{vDBB}+\mathrm{NBM}$ groups and showed a clear trend $(p<0.10)$ in the VTA + MS/vDBB group (Fig. $3 C$ ). It should be noted also that in a separate analysis we found that all of the VTA lesion groups operated at chance level (i.e., not different from $15 \mathrm{~s}$ time allocation in each of the quadrants; one-sample $t$ test, $p>0.05$ for all comparisons in the VTA groups).

\section{Combined loss of dopaminergic and cholinergic inputs to the hippocampus is sufficient to induce a working memory deficit}

The working memory version of the MWM test was administered on completion of the reference memory task. In this design, the platform was moved to a new quadrant everyday over 4 consecutive days. The animals were given four trials with $20 \mathrm{~s}$ intertrial time on each testing day forcing them to relearn the new platform position within the four trials. The performances of the groups and the relevant statistics are shown in Figure 4 and its legend. All animals exhibited in general a long latency to find the submerged platform on the first trial of each day, but once the platform position was identified, normal rats were able to locate it within progressively shorter times and showed no further improvements between the third and the fourth trial (Fig. $4 A$ ). To provide a measure of the learning efficiency in this task, the percentage improvement between trials 1 and 2 (Fig. 4B) and between trials 1 and 3 (Fig. 4C) were analyzed and plotted in terms of savings (Netto et al., 1993). Under these conditions, control rats were seen to reduce their latency to reach the platform by $\sim 50-70 \%$. The cholinergic or 
dopaminergic lesions alone did not induce any measurable deficit in this test. However, when the dopaminergic lesion was combined with the lesion of the septohippocampal projection (VTA $+\mathrm{MS} / \mathrm{vDBB}$ group), there was a clear worsening in working memory performance (Fig. $4 B, C)$. This effect appeared to be mediated by impairment at the hippocampal rather than the frontal cortex level, because combined lesions of the VTA and NBM were ineffective, and lesioning both cholinergic subsystems and the VTA (triple lesion group) did not provide any additional impairment beyond that exhibited by the VTA+MS/vDBB group.

\section{Working memory deficits in the} combined DA- and ACh-lesioned animals are attributable to inability to develop an efficient search strategy

The reference memory deficits and the apparently normal working memory in the VTA-lesioned animals, as opposed to a dual impairment seen in the combined VTA- and MS/vDBB-lesioned group, suggested that the two neurotransmitter systems were likely to provide critical regulatory inputs to different aspects of memory performance. To explain the nature of the deficits in these groups, the strategy used to find the platform during the working memory test was closely inspected (Fig. 5).

Thus, the swim paths recorded from control, VTA-lesioned, and VTA+MS/ vDBB-lesioned animals during the execution of the working memory task were evaluated by a blinded observer and allocated into one of the following categories: (1) use of spatial strategy, (2) use of nonspatial strategy, and (3) no clear-cut strategy as detailed in Materials and Methods. Normal control animals predominantly used a spatial strategy previously in the first trial and used almost exclusively this approach also during the subsequent trials (Fig. $5 A, B$ ). In contrast, several animals in both the VTA and the VTA+MS/vDBB groups showed no or poor search strategy in the first trial (effect of groups, Pearson $\chi_{(847,4)}^{2}=172.87, p<0.0001$; effect of trials, Pearson $\chi_{(847,6)}^{2}=61.46, p<$ 0.0001 ; effect of days, Pearson $\chi_{(847,6)}^{2}=$ 2.39, $p=0.88$ ) (Fig. $5 D, F)$. They, however, differed markedly from each other in the strategy exhibited in the subsequent trials within the same testing day. The VTA-lesioned animals progressively used a spatial or nonspatial strategy, with a pattern that was similar in all four testing days. This suggests that DAdepleted rats were unable to retain information of the search strategy from the previous testing day, although they were capable of learning the platform position and use extra maze cues with repeated trials on the same day (Fig. 5C,D). The VTA + MS/vDBB

\section{A Reference memory task}
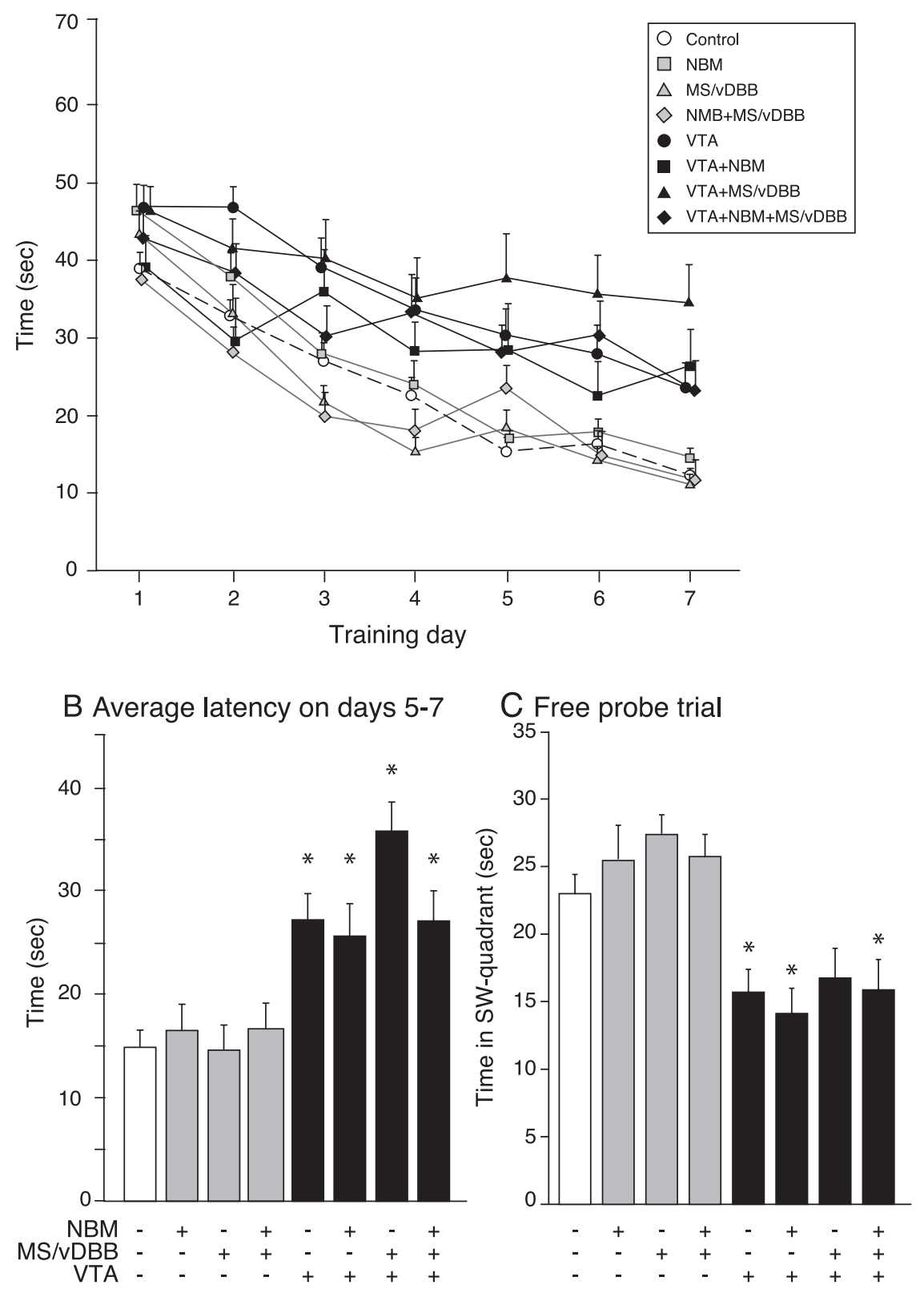

Figure 3. Reference memory performance in the Morris water maze task. $\boldsymbol{A}-\boldsymbol{C}$, Data are illustrated as average latencies over testing days $(\boldsymbol{A})$, average latency of days $5-7$ across groups $(\boldsymbol{B})$, and the free probe test $(\boldsymbol{C})$. $\boldsymbol{A}$, Control animals showed a decrease in latency time over the training days. $\boldsymbol{A}, \boldsymbol{B}$, Lesioning the cholinergic projection neurons in the NBM, MS/vDBB, or in both structures did not affect the learning capabilities of the rats. However, lesioning the dopaminergic neurons in the VTA resulted in disruption of the performance in these animals ( $\boldsymbol{A}$, filled symbols). $\boldsymbol{B}$, Comparison of the performance between the groups on the average latency scores on days 5 -7 when an asymptote was established in the control group showed that all VTA-lesioned groups took longer to find the platform to control animals. $C$, The free probe test confirmed that loss of dopaminergic neurons in the VTA resulted in a less-focused search for the platform, as shown by reduction in time spent in the target quadrant. Data are shown as mean latency values \pm SEM. Difference compared with control group at ${ }^{*} p<0.05\left(\boldsymbol{B}\right.$ : 1-way ANOVA, $F_{(7,109)}=9.32, p<0.0001$, followed by post hoc Tukey HSD tests; $C$ : 1-way ANOVA, $F_{(7,109)}=7.48, p<0.0001$, followed by post hoc Tukey HSD tests). $B, C$, Groups are represented in the $x$-axis by presence $(+)$ or absence $(-)$ of a lesion in the given nucleus. Error bars indicate SEM.

group, however, exhibited an overall poor performance across all trials. Approximately $50 \%$ of the animals failed to develop a strategy despite repeated training (Fig. $5 E, F$ ). Therefore, the inability to develop an efficient search strategy appeared to be a distinctive behavior in most of the animals with such combined lesions. These results suggested also that the apparently similar impairments seen in the VTA and VTA+MS/vDBB groups in the refer- 


\section{A Working memory task}

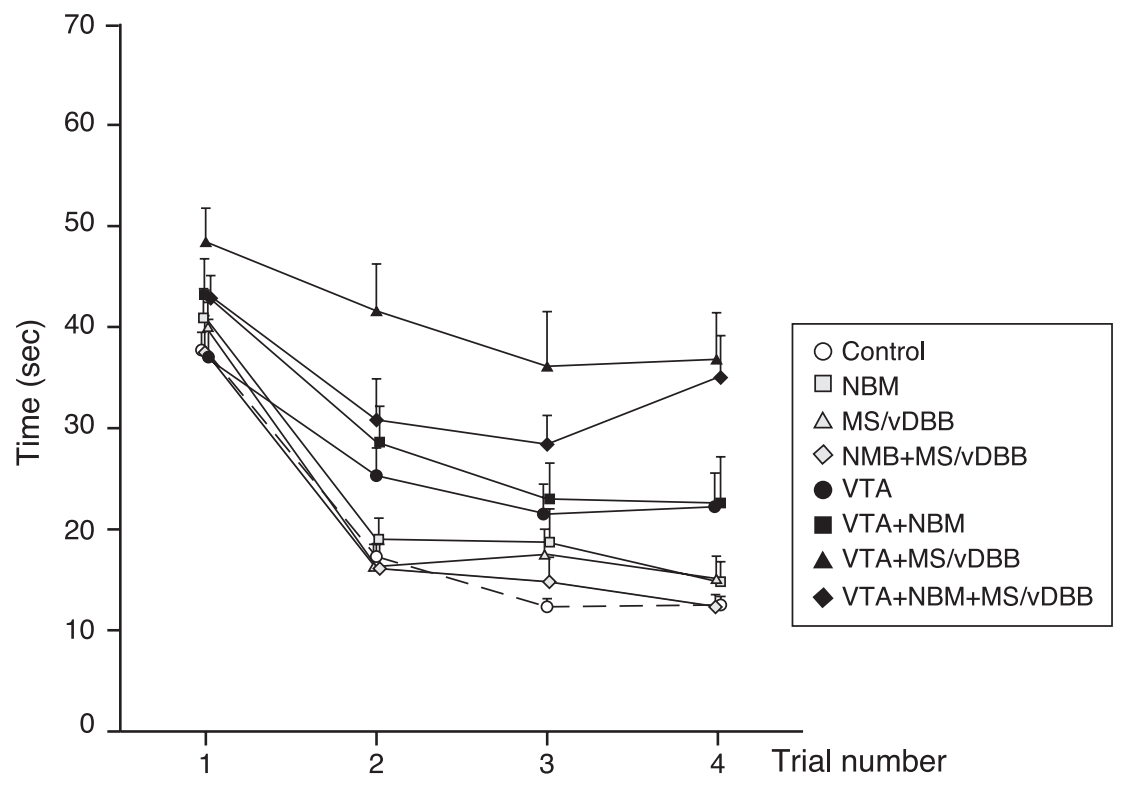

Improvement in latency
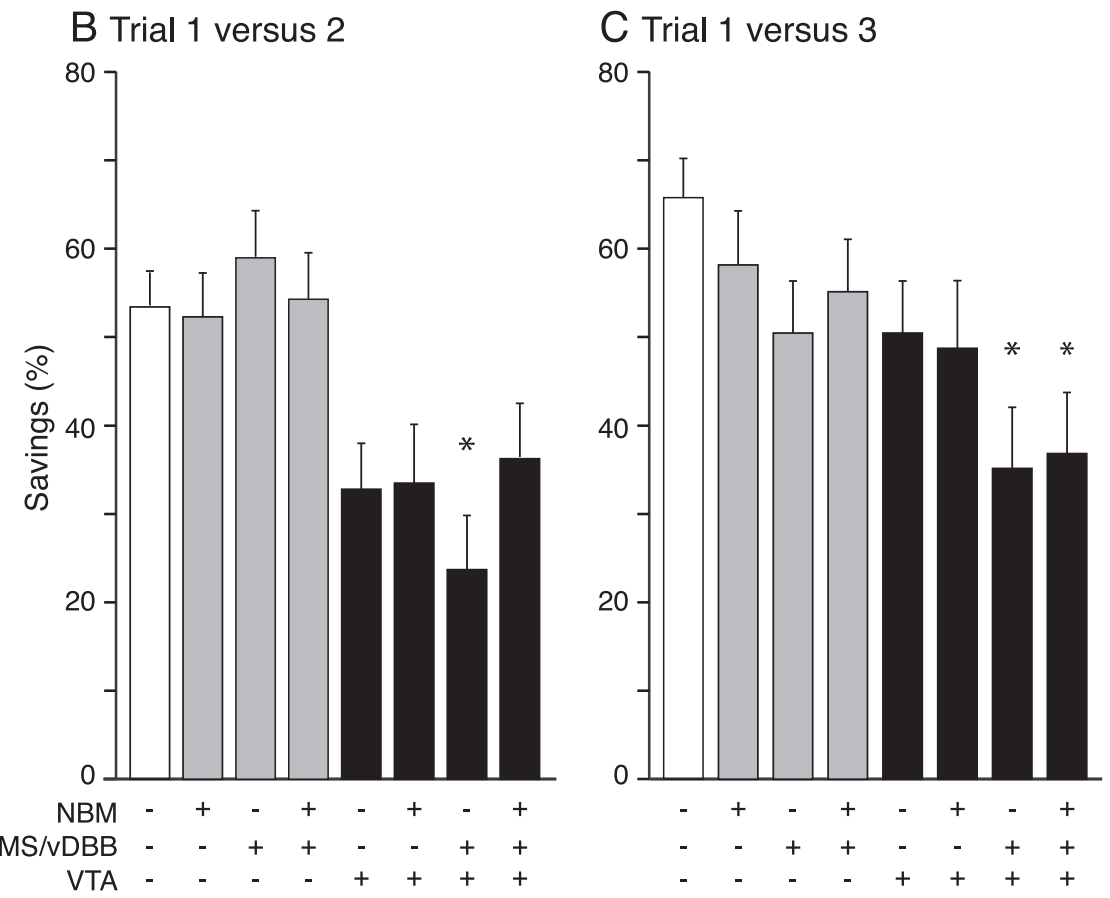

Figure 4. Working memory performance in the Morris water maze task. $A-C$, Data are illustrated as average latency to find the platform in consecutive trials $(\boldsymbol{A})$ and percentage improvement between trials 1 and $2(\boldsymbol{B})$, and trials 1 and $3(\boldsymbol{C})$. $\boldsymbol{A}$, Control animals showed a decrease in latency over the four trials, reaching a plateau performance in trials 3 and 4. $B, C$, Although lesioned groups showed a slight decrease in the savings measure compared with the control group, only animals with a combined lesion of dopaminergic neurons in the VTA and cholinergic neurons in the MS/vDBB resulted in a significant worsening. Lesioning cholinergic neurons in the NBM did not increase the deficits seen in the double-lesioned rats. Data shown are means \pm SEM. Difference compared with control group at ${ }^{*} p<0.05$ (B: 1-way ANOVA, $F_{(7,109)}=4.82, p<0.0001$, followed by post hoc Tukey HSD tests; C: 1 -way ANOVA, $F_{(7,109)}=5.51, p<0.0001$, followed by post hoc Tukey HSD tests). $B, C$, Groups are represented in the $x$-axis by presence $(+)$ or absence $(-)$ of a lesion in the given nucleus. Error bars indicate SEM.

ence memory test might have been different in nature. To explore this possibility, we analyzed the reference memory data on a trialby-trial basis (Fig. 6). In addition to the VTA and VTA+MS/ vDBB groups, normal controls and MS/vDBB-lesioned animals were also included in this analysis for comparison. On the first testing day, the latency to find the platform was similar in all four groups. On days $2-4$, control animals quickly improved their performance with repeated trials but required a relatively longer latency on the first trial. In the following days, the control and the MS/vDBB groups reached a steady performance, with similar latency scores between the last and first trial of 2 consecutive days. Conversely, and despite an improvement in latency in each training day, VTA-lesioned animals exhibited latencies in the first trials that remained higher than those recorded during the last trials of the previous training day. Thus, unlike the control, the VTA-lesioned group was unable to consolidate the learned information and had to systematically relearn the platform position. It should be noted here that in addition to a mnemonic impairment, altered anxiety and/or exploratory behavior could have contributed to the memory deficiency in these animals. As we have not directly measured anxiety in these animals, from the present data, we cannot determine whether altered anxiety might have contributed to the memory deficits seen here.

Finally, the learning impairments in these animals appeared to be specific for hippocampus-dependent spatial learning tasks as none of the groups showed any detectable deficits in a striatumdependent motor learning task in the staircase test (Fig. 7). All animals learned the fine motor skills needed to successfully retrieve the food pellets. On the first day of training the rats reached an average of 48 of 80 pellets (termed as attempts) presented to them on each side of a central platform and were able to successfully retrieve $\sim 23$ pellets. After $5 \mathrm{~d}$ of training, all animals improved their success rates and reached a plateau level in performance, both in the number of attempts (a mean of 76 of 80 pellets across groups) and successes (a mean of 61 pellets). When the numbers of errors (defined as the number of pellets missed as percentage of total number of attempts) were compared, no difference was observed across groups at any of the testing days.

\section{Discussion}

The aim of the present study was to examine the importance of the mesocorticolimbic DA and the septohippocampal and basalocortical cholinergic projections in the regulation of aspects of learning and memory using a wellestablished swim maze task. We hypothesized that a functional interaction between these two modulatory neurotransmitter sys- 
tems may be critical in maintaining normal memory performances in the rat. We found that lesioning the cholinergic neurons in the BF by direct intraparenchymal injections of the $192 \mathrm{IgG}$-saporin toxin did not affect the rats' ability to perform in the versions of the MWM task used here. However, disruption of the mesocorticolimbic DA pathway by injection of the 6-OHDA toxin was sufficient to induce marked acquisition deficits in the reference but not in the working memory version of the MWM task. Importantly, a combined lesion of the dopaminergic neurons in the VTA and the cholinergic neurons in the MS/vDBB was sufficient to impair the animals' performance in the working memory task. This finding suggests that integrity of both the DA and ACh regulatory inputs to the hippocampus may be critical for working memory processing. The basalo-cortical cholinergic projection does not appear to be involved in such regulation. In fact, lesioning NBM neurons did not produce any clear-cut effects either alone or when combined with dopaminergic lesion in the VTA. This applied both to the cognitive functions and to the ability to learn motor skills. The latter function was previously shown to be impaired in a different test paradigm (Conner et al., 2003). Together, these findings suggest that the functional convergence of ACh and DA systems mediate the actual learning in hippocampaldependent short-term memory tasks, whereas the DA system has in addition a critical role in the consolidation of the learned strategies.

Studies in rodents and primates have implicated the hippocampal and neocortical regions in learning and memory functions. The hippocampus, in particular, is thought to be required in the initial encoding of spatial memories and their retrieval after short delays (Squire, 1992; Alvarez and Squire, 1994). However, it may not play a central role in long-term storage of the spatial memory information. According to this standard consolidation theory, the hippocampus is involved in the initial linking or indexing of the memory representations in the neocortical regions, but with time, memory representations can be consolidated. In this way, the neocortical regions can become the primary site activated for execution of the learned memory tasks, and in some (nonspatial) tasks they may even function independent of the hippocampus (Frankland and Bontempi, 2005; Spiers and Maguire, 2007). This can be seen in experimental paradigms in that silencing the hippocampus causes disruption in the retrieval of recent

E

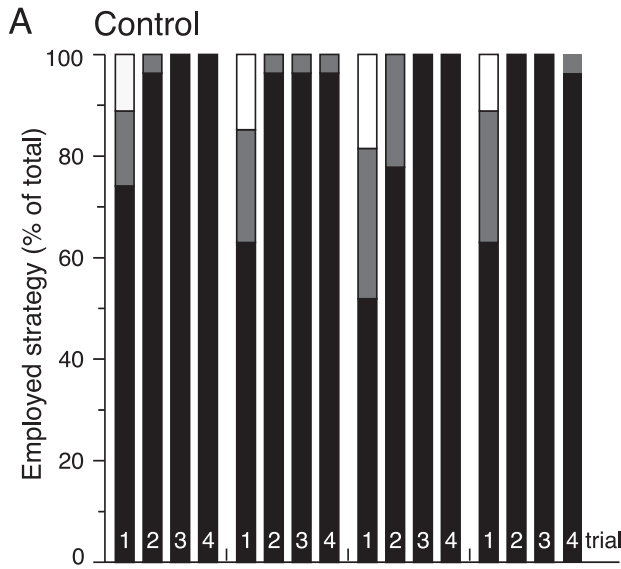

B
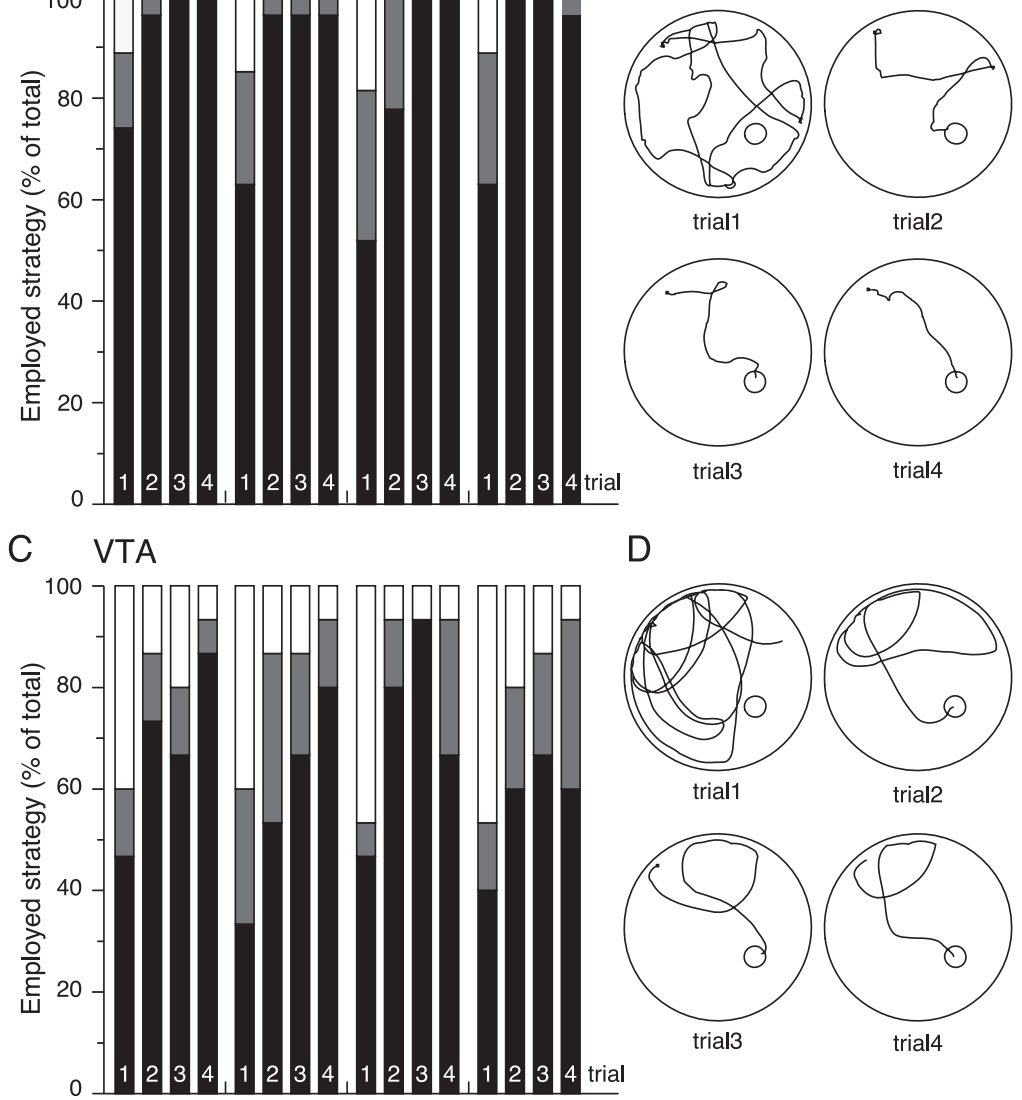

.
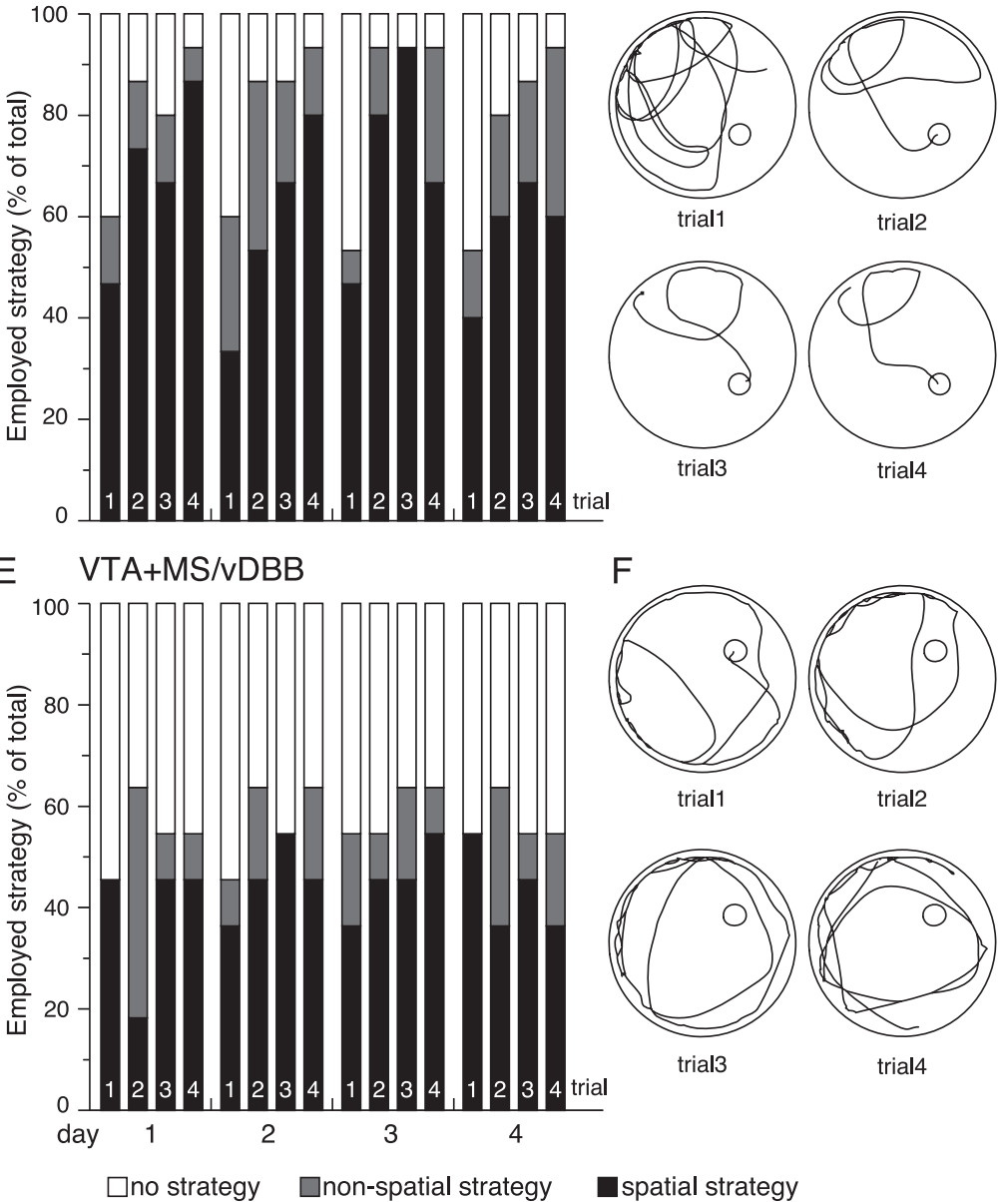

spatial strategy

Figure 5. Search strategy used during the working memory version of the MWM test. $\boldsymbol{A}-\boldsymbol{F}$, Scores from three groups of animals (controls, VTA, and VTA + MS/vDBB) are illustrated as bar graphs $(\boldsymbol{A}, \boldsymbol{C}, \boldsymbol{E})$ and sample swim paths from representative animals in the respective groups $(\boldsymbol{B}, \boldsymbol{D}, \boldsymbol{F}) . \boldsymbol{A}, \boldsymbol{B}$, Almost all animals in the control group showed a clearly recognized search strategy in all four trials on each of the four testing days. $\boldsymbol{A}$, Most animals adapted a spatial search strategy and were able to locate the platform quickly. $\boldsymbol{B}$, Note that the example trace shown for trial one in this group illustrates that the rat is searching the platform position in the entire area of the pool, but once the new position is found, it rapidly adapts to this and orients toward the platform very effectively in the following trials. $\boldsymbol{C}, \boldsymbol{D}$, The VTA-lesioned animals differed from the controls in that they performed rather poorly in the first trial on each day (i.e., $\sim 50 \%$ of the animals showed no strategy) but dramatically improved with repeated trials to reach a performance similar to that of the intact animals. $\boldsymbol{E}, \boldsymbol{F}$, Approximately $60 \%$ of the animals in the VTA + MS/vDBB group, however, were not able to use a spatial or nonspatial search strategy even with repeated trials.

but not of remote memories. In contrast, when the frontal or the anterior cingulate cortex is inactivated, retrieval of remote memories can be impaired (Maviel et al., 2004).

In our experimental paradigm, lesioning of the dopaminergic 


\section{Reference memory task \\ (trial by trial breakdown of latency)}

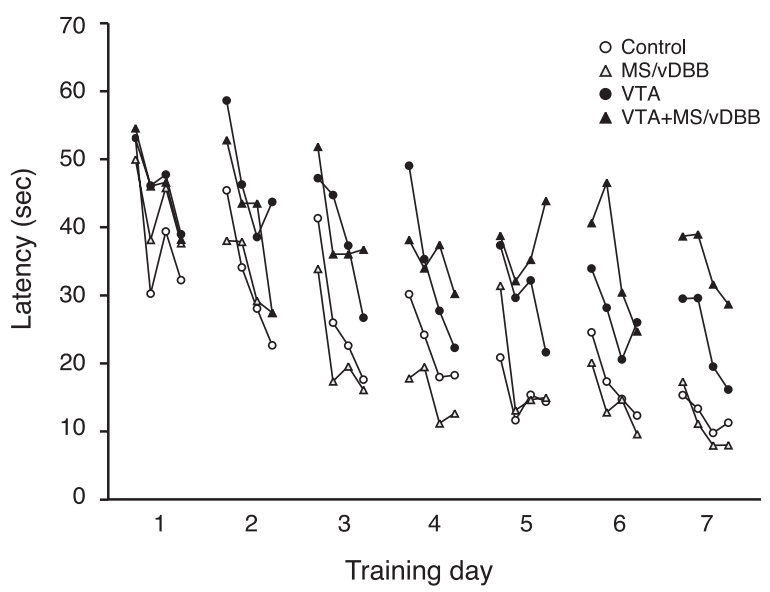

Figure 6. Reference memory performance in the MWM test. A trial-by-trial breakdown of the data are illustrated for four experimental groups. Control and MS/VDBB-lesioned animals behaved similarly and decreased their latency to find the platform, and from day 5 onward showed a steady performance both within and between the training days. The VTA group was capable of improving the time to find the platform within one training day; however, they did not seem to be able to retain this information to the next training day.

\section{Paw reaching test}

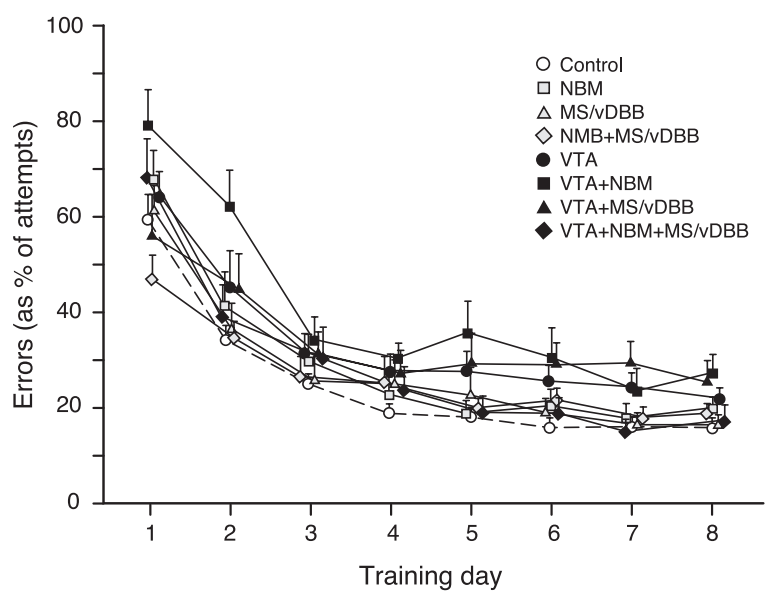

Figure 7. Striatum-dependent motor learning was tested using the paw-reaching task in the staircase test. The data are illustrated in errors as percentage of total attempts. All groups reduced the amount of errors made over time (2-way repeated-measures ANOVA, effect of time, $\left.F_{(7,109)}=46.8, p<0.0001\right)$, but no statistically significant difference was found in the overall analysis (2-way repeated-measures ANOVA, group $\times$ time interaction, $F_{(7,109)}=0.86$, $p=0.73)$. Error bars indicate SEM.

mesocorticolimbic pathway caused reference memory impairment in the MWM task. Although, from this data, it is not possible to conclude whether the deficit is in the encoding or the storage/recall of the learned position of the platform; the fact that these lesioned animals performed as efficiently as the normal controls in the working memory task argues that the latter explanation is more plausible. The difference between these two versions of the MWM test is that the position of the platform changes everyday in the working memory task, forcing the animals to acquire and employ new orienting cues within minutes during the test session, whereas in the reference memory task, the animals were trained for $7 \mathrm{~d}$ to learn a fixed platform position, in which consolidation of learned memories has an impact on the final performance of the animal. The interpretation that a partial lesion of the mesocorticolimbic DA pathway resulted in a consolidation, but not in an encoding deficit, became clearer on closer examination of the swim paths in the working memory task. Although approximately one-half of the animals in the VTA group did not have a search strategy in the first trial of the working memory test sessions, they were able to adapt a strategy and locate the platform faster with repeated training. However, they seemed to be incapable of carrying this information to the next day. A similar pattern was evident in the trial-by-trial analysis of the latencies in the reference memory task. The difference in latency between the last and the first trials of two consecutive training days was much higher in the VTA compared with control group on days $5-7$ when consolidation was previously achieved by the intact rats. The control animals clearly improved their average latency to find the platform, compared with the VTA group, suggesting that they could recall the position of the platform from the previous day. However, the consolidation advantage could not be used in the working memory task as the platform position changed everyday. Thus, this testing paradigm yielded similar performance levels in both normal intact and DAdepleted rats as measured by savings between trials 1 and 2 or trials 1 and 3 on the same day.

Accumulating data suggest that the entry of new information into long-term memory is controlled by a VTA-hippocampus loop (for review, see Lisman and Grace, 2005). Dopaminergic projections originating from the VTA innervate the CA1 field and subiculum (Verney et al., 1985; Gasbarri et al., 1997). When rats were exposed to novel stimuli, dopaminergic neurons in the VTA respond by increased firing (Ljungberg et al., 1992). This results in an increased release of DA in the hippocampus (Ihalainen et al., 1999), which is thought to be important in the late stage of long-term potentiation (LTP) in the CA1 region of the hippocampus (Frey et al., 1991). In addition, it has been demonstrated that a novel environment can lower the threshold for hippocampal LTP induction, which could be blocked by a D1/ D5-receptor antagonist (Li et al., 2003). However, consolidation of learned tasks can also be mediated by DA inputs at the cortical level. In fact, blocking the D1-receptor activity in the medial frontal cortex has been observed to inhibit long-term memory formation (Izquierdo et al., 2007). Interestingly, the CA1 region and the subiculum are known to be connected to the medial frontal cortex via a direct (monosynaptic) pathway [for review, see Laroche et al. (2000)]. Together, these data suggest that the behavioral deficits exhibited by the VTA lesion group in the present study could be indirectly attributable to an alteration in the activity of the hippocampo-cortical projections and/or a direct effect of DA loss in the relevant cortical regions. Whether the hippocampo-striatal connections play a role in these deficits cannot be determined definitely because the test paradigms used here do not allow for this distinction.

Ascending cholinergic inputs have been involved in spatial learning and memory, although the claim of their essential role in cognition has recently been called into question (for review, see Parent and Baxter, 2004). The amnestic properties of anticholinergic drugs such as scopolamine and atropine are well documented (Collerton, 1986), and it has also been shown that ACh levels in the hippocampus increase during the execution of hippocampus-dependent memory tasks (Yamamuro et al., 1995; Chang and Gold, 2003). However, more selective lesions of the septohippocampal pathway by 192 IgG-saporin have failed to replicate the behavioral impairments seen after cholinergic pharmacological blockade or nonspecific lesions (McMahan et al., 
1997; Fletcher et al., 2007). This would suggest that in these drugor lesion-induced effects on behavior, other cholinergic neurons outside the BF and/or noncholinergic populations in other transmitter systems might participate as well. Thus, the specific role of the septohippocampal cholinergic projections in the regulation of memory formation remained elusive and a matter for debate. Here, we found that depletion of DA and ACh at the level of the hippocampus by combined lesions of the dopaminergic neurons in the VTA and cholinergic neurons in the MS/vDBB induced a significant deficit in the acquisition of new information in a working memory task. Because lesioning either neuronal systems alone did not impair the animals' performance in this task, the data argue that the concurrent disruption of the dopaminergic and cholinergic inputs at the level of the hippocampus is necessary for induction of acquisition deficits in the rat. Thus, a functional convergence of the two modulatory neurotransmitter systems appears to be critical for normal execution of hippocampusdependent working memory tasks.

Cognitive deficits are common in PD patients and might even be observed in newly diagnosed cases. Tasks that require visual analysis and orientation, as well as working memory performance may be particularly compromised (Levin et al., 1991; Dujardin and Laurent, 2003). Although some patients might show impairments only in frontostriatal or temporal lobe functions, others might demonstrate more global deficits as studied using the Mini-Mental State Examination, a pattern recognition task, and the Tower of London task (Foltynie et al., 2004). Imaging studies performed in PD patients while performing on working memory tasks support the interpretation that VTA dopaminergic projection facilitates memory functions via direct inputs to the prefrontal cortex (Cools et al., 2002; Mattay et al., 2002). In light of the present results, it appears plausible that a partial $(\approx 45 \%)$ loss of dopaminergic cells in the VTA and a corresponding 60$70 \%$ depletion of the DA content in the prefrontal cortex and the hippocampus, as seen in PD (Scatton et al., 1983; Damier et al., 1999), could readily explain some of the cognitive deficits in these patients. Nevertheless, a concomitant degeneration of the BF cholinergic systems, especially in the later stages of the disease, could worsen the cognitive symptoms (Calabresi et al., 2006). Therefore, further analysis of cholinergic alterations in PD patients may help to understand and treat the nonmotor symptoms of the disease.

\section{References}

Aarsland D, Andersen K, Larsen JP, Lolk A, Kragh-Sørensen P (2003) Prevalence and characteristics of dementia in Parkinson disease: an 8-year prospective study. Arch Neurol 60:387-392.

Agid YA, Javoy-Agid F, Ruberg M (1987) Biochemistry of neurotransmitters in Parkinson's disease. In: Movement disorders 2 (Marsden CD, Fahn S, eds), pp 166-230. London: Butterworths.

Alvarez P, Squire LR (1994) Memory consolidation and the medial temporal lobe: a simple network model. Proc Natl Acad Sci U S A 91:7041-7045.

Bartus RT, Dean RL 3rd, Beer B, Lippa AS (1982) The cholinergic hypothesis of geriatric memory dysfunction. Science 217:408-414.

Bigl V, Woolf NJ, Butcher LL (1982) Cholinergic projections from the basal forebrain to frontal, parietal, temporal, occipital, and cingulate cortices: a combined fluorescent tracer and acetylcholinesterase analysis. Brain Res Bull 8:727-749.

Braak H, Rüb U, Jansen Steur EN, Del Tredici K, de Vos RA (2005) Cognitive status correlates with neuropathologic stage in Parkinson disease. Neurology 64:1404-1410.

Breysse N, Carlsson T, Winkler C, Björklund A, Kirik D (2007) The functional impact of the intrastriatal dopamine neuron grafts in parkinsonian rats is reduced with advancing disease. J Neurosci 27:5849-5856.

Calabresi P, Picconi B, Parnetti L, Di Filippo M (2006) A convergent model for cognitive dysfunctions in Parkinson's disease: the critical dopamineacetylcholine synaptic balance. Lancet Neurol 5:974-983.

Cassel JC, Gaurivaud M, Lazarus C, Bertrand F, Galani R, Jeltsch H (2002) Grafts of fetal septal cells after cholinergic immunotoxic denervation of the hippocampus: a functional dissociation between dorsal and ventral implantation sites. Neuroscience 113:871-882.

Chang Q, Gold PE (2003) Switching memory systems during learning: changes in patterns of brain acetylcholine release in the hippocampus and striatum in rats. J Neurosci 23:3001-3005.

Collerton D (1986) Cholinergic function and intellectual decline in Alzheimer's disease. Neuroscience 19:1-28.

Conner JM, Culberson A, Packowski C, Chiba AA, Tuszynski MH (2003) Lesions of the Basal forebrain cholinergic system impair task acquisition and abolish cortical plasticity associated with motor skill learning. Neuron 38:819-829.

Cools R, Stefanova E, Barker RA, Robbins TW, Owen AM (2002) Dopaminergic modulation of high-level cognition in Parkinson's disease: the role of the prefrontal cortex revealed by PET. Brain 125:584-594.

Coyle JT, Price DL, DeLong MR (1983) Alzheimer's disease: a disorder of cortical cholinergic innervation. Science 219:1184-1190.

Dagher A, Owen AM, Boecker H, Brooks DJ (2001) The role of the striatum and hippocampus in planning: a PET activation study in Parkinson's disease. Brain 124:1020-1032.

Damier P, Hirsch EC, Agid Y, Graybiel AM (1999) The substantia nigra of the human brain. II. Patterns of loss of dopamine-containing neurons in Parkinson's disease. Brain 122:1437-1448.

Dubois B, Ruberg M, Javoy-Agid F, Ploska A, Agid Y (1983) A subcorticocortical cholinergic system is affected in Parkinson's disease. Brain Res 288:213-218.

Dujardin K, Laurent B (2003) Dysfunction of the human memory systems: role of the dopaminergic transmission. Curr Opin Neurol 16 [Suppl 2]:S11-S16.

Fearnley JM, Lees AJ (1991) Ageing and Parkinson's disease: substantia nigra regional selectivity. Brain 114:2283-2301.

Fischer W, Gage FH, Björklund A (1989) Degenerative changes in forebrain cholinergic nuclei correlate with cognitive impairments in aged rats. Eur J Neurosci 1:34-45.

Fletcher BR, Baxter MG, Guzowski JF, Shapiro ML, Rapp PR (2007) Selective cholinergic depletion of the hippocampus spares both behaviorally induced Arc transcription and spatial learning and memory. Hippocampus 17:227-234.

Foltynie T, Brayne C, Barker RA (2002) The heterogeneity of idiopathic Parkinson's disease. J Neurol 249:138-145.

Foltynie T, Brayne CE, Robbins TW, Barker RA (2004) The cognitive ability of an incident cohort of Parkinson's patients in the UK. The CamPaIGN study. Brain 127:550-560.

Frankland PW, Bontempi B (2005) The organization of recent and remote memories. Nat Rev Neurosci 6:119-130.

Frey U, Matthies H, Reymann KG, Matthies H (1991) The effect of dopaminergic D1 receptor blockade during tetanization on the expression of long-term potentiation in the rat CA1 region in vitro. Neurosci Lett 129:111-114.

Gasbarri A, Sulli A, Packard MG (1997) The dopaminergic mesencephalic projections to the hippocampal formation in the rat. Prog Neuropsychopharmacol Biol Psychiatry 21:1-22.

Gundersen HJ, Bendtsen TF, Korbo L, Marcussen N, Møller A, Nielsen K, Nyengaard JR, Pakkenberg B, Sørensen FB, Vesterby A, West MJ (1988) Some new, simple and efficient stereological methods and their use in pathological research and diagnosis. Apmis 96:379-394.

Hirsch E, Graybiel AM, Agid YA (1988) Melanized dopaminergic neurons are differentially susceptible to degeneration in Parkinson's disease. Nature 334:345-348.

Ihalainen JA, Riekkinen P Jr, Feenstra MG (1999) Comparison of dopamine and noradrenaline release in mouse prefrontal cortex, striatum and hippocampus using microdialysis. Neurosci Lett 277:71-74.

Izquierdo LA, Barros DM, da Costa JC, Furini C, Zinn C, Cammarota M, Bevilaqua LR, Izquierdo I (2007) A link between role of two prefrontal areas in immediate memory and in long-term memory consolidation. Neurobiol Learn Mem 88:160-166.

Jog MS, Kubota Y, Connolly CI, Hillegaart V, Graybiel AM (1999) Building neural representations of habits. Science 286:1745-1749.

Kirik D, Rosenblad C, Björklund A (1998) Characterization of behavioral 
and neurodegenerative changes following partial lesions of the nigrostriatal dopamine system induced by intrastriatal 6-hydroxydopamine in the rat. Exp Neurol 152:259-277.

Laroche S, Davis S, Jay TM (2000) Plasticity at hippocampal to prefrontal cortex synapses: dual roles in working memory and consolidation. Hippocampus 10:438-446.

Leanza G, Nilsson OG, Wiley RG, Björklund A (1995) Selective lesioning of the basal forebrain cholinergic system by intraventricular 192 IgG-saporin: behavioural, biochemical and stereological studies in the rat. Eur J Neurosci 7:329-343.

Lehmann J, Nagy JI, Atmadia S, Fibiger HC (1980) The nucleus basalis magnocellularis: the origin of a cholinergic projection to the neocortex of the rat. Neuroscience 5:1161-1174.

Lehmann O, Jeltsch H, Lehnardt O, Pain L, Lazarus C, Cassel JC (2000) Combined lesions of cholinergic and serotonergic neurons in the rat brain using $192 \mathrm{IgG}$-saporin and 5,7-dihydroxytryptamine: neurochemical and behavioural characterization. Eur J Neurosci 12:67-79.

Levin BE, Llabre MM, Reisman S, Weiner WJ, Sanchez-Ramos J, Singer C, Brown MC (1991) Visuospatial impairment in Parkinson's disease. Neurology 41:365-369.

Lewis SJ, Dove A, Robbins TW, Barker RA, Owen AM (2003) Cognitive impairments in early Parkinson's disease are accompanied by reductions in activity in frontostriatal neural circuitry. J Neurosci 23:6351-6356.

Lewis SJ, Foltynie T, Blackwell AD, Robbins TW, Owen AM, Barker RA (2005) Heterogeneity of Parkinson's disease in the early clinical stages using a data driven approach. J Neurol Neurosurg Psychiatry 76:343-348.

Li S, Cullen WK, Anwyl R, Rowan MJ (2003) Dopamine-dependent facilitation of LTP induction in hippocampal CA1 by exposure to spatial novelty. Nat Neurosci 6:526-531.

Lisman JE, Grace AA (2005) The hippocampal-VTA loop: controlling the entry of information into long-term memory. Neuron 46:703-713.

Ljungberg T, Apicella P, Schultz W (1992) Responses of monkey dopamine neurons during learning of behavioral reactions. J Neurophysiol 67:145-163.

Maingay M, Romero-Ramos M, Carta M, Kirik D (2006) Ventral tegmental area dopamine neurons are resistant to human mutant alpha-synuclein overexpression. Neurobiol Dis 23:522-532.

Marder K, Tang MX, Cote L, Stern Y, Mayeux R (1995) The frequency and associated risk factors for dementia in patients with Parkinson's disease. Arch Neurol 52:695-701.

Mattay VS, Tessitore A, Callicott JH, Bertolino A, Goldberg TE, Chase TN, Hyde TM, Weinberger DR (2002) Dopaminergic modulation of cortical function in patients with Parkinson's disease. Ann Neurol 51:156-164.

Maviel T, Durkin TP, Menzaghi F, Bontempi B (2004) Sites of neocortical reorganization critical for remote spatial memory. Science 305:96-99.

McMahan RW, Sobel TJ, Baxter MG (1997) Selective immunolesions of hippocampal cholinergic input fail to impair spatial working memory. Hippocampus 7:130-136.

Montoya CP, Campbell-Hope LJ, Pemberton KD, Dunnett SB (1991) The "staircase test": a measure of independent forelimb reaching and grasping abilities in rats. J Neurosci Methods 36:219-228.

Nakano I, Hirano A (1984) Parkinson's disease: neuron loss in the nucleus basalis without concomitant Alzheimer's disease. Ann Neurol 15:415-418.

Netto CA, Hodges H, Sinden JD, Le Peillet E, Kershaw T, Sowinski P, Meldrum BS, Gray JA (1993) Effects of fetal hippocampal field grafts on ischaemic-induced deficits in spatial navigation in the water maze. Neuroscience 54:69-92.

Owen AM, Doyon J, Dagher A, Sadikot A, Evans AC (1998) Abnormal basal ganglia outflow in Parkinson's disease identified with PET. Implications for higher cortical functions. Brain 121:949-965.

Parent MB, Baxter MG (2004) Septohippocampal acetylcholine: involved in but not necessary for learning and memory? Learn Mem 11:9-20.

Paxinos G, Watson C (2007) The rat brain in stereotaxic coordinates, Ed 6. San Diego: Elsevier.

Scatton B, Javoy-Agid F, Rouquier L, Dubois B, Agid Y (1983) Reduction of cortical dopamine, noradrenaline, serotonin and their metabolites in Parkinson's disease. Brain Res 275:321-328.

Spiers HJ, Maguire EA (2007) The neuroscience of remote spatial memory: a tale of two cities. Neuroscience 149:7-27.

Squire LR (1992) Memory and the hippocampus: a synthesis from findings with rats, monkeys, and humans. Psychol Rev 99:195-231.

Torres EM, Perry TA, Blockland A, Wilkinson LS, Wiley RG, Lappi DA, Dunnet SB (1994) Behavioural, histochemical and biochemical consequences of selective immunolesions in discrete regions of the basal forebrain cholinergic system. Neuroscience 63:95-122.

Torres EM, Meldrum A, Kirik D, Dunnett SB (2006) An investigation of the problem of two-layered immunohistochemical staining in paraformaldehyde fixed sections. J Neurosci Methods 158:64-74.

Verney C, Baulac M, Berger B, Alvarez C, Vigny A, Helle KB (1985) Morphological evidence for a dopaminergic terminal field in the hippocampal formation of young and adult rat. Neuroscience 14:1039-1052.

West MJ (1999) Stereological methods for estimating the total number of neurons and synapses: issues of precision and bias. Trends Neurosci 22:51-61.

West MJ, Slomianka L, Gundersen HJ (1991) Unbiased stereological estimation of the total number of neurons in the subdivisions of the rat hippocampus using the optical fractionator. Anat Rec 231:482-497.

White NM (1997) Mnemonic functions of the basal ganglia. Curr Opin Neurobiol 7:164-169.

Williams-Gray CH, Foltynie T, Brayne CE, Robbins TW, Barker RA (2007) Evolution of cognitive dysfunction in an incident Parkinson's disease cohort. Brain 130:1787-1798.

Woolf NJ (1991) Cholinergic systems in mammalian brain and spinal cord. Prog Neurobiol 37:475-524.

Yamamuro Y, Hori K, Tanaka J, Iwano H, Nomura M (1995) Septohippocampal cholinergic system under the discrimination learning task in the rat: a microdialysis study with the dual-probe approach. Brain Res 684:1-7. 